\title{
Eficacia del Método Flipped Classroom en la Universidad: Meta-Análisis de la Producción Científica de Impacto
}

\author{
Efficacy of the Flipped Classroom Method at the University: \\ Meta-Analysis of Impact Scientific Production
}

\author{
Tomás Sola Martínez * \\ Inmaculada Aznar Díaz \\ José María Romero Rodríguez \\ Antonio-Manuel Rodríguez-García \\ Universidad de Granada, España
}

\begin{abstract}
La particularidad del método flipped classroom se halla en el cambio de los roles de aprendizaje, donde el profesorado adquiere un rol secundario como guía y el estudiante un rol primario como agente activo. En esta línea, la tendencia en su implementación en la etapa de educación superior va en aumento y cada vez es más habitual escuchar experiencias docentes que giran en torno a su aplicación en el aula. Por ello, este trabajo tiene como objetivo analizar el efecto de la metodología flipped classroom en el rendimiento académico de los estudiantes universitarios a partir de los estudios indexados en las bases de datos wos y Scopus. Se ha seguido una metodología propia de los estudios de meta-análisis, con la finalidad de medir el tamaño del efecto global y particular de las investigaciones analizadas y constatar empíricamente la ganancia a favor del grupo experimental o control. Los resultados muestran que la diferencia entre grupos es estadísticamente significativa y la mayor parte de los estudios confirma el aumento del rendimiento académico en el grupo experimental. No obstante, se evidencia un posible sesgo de los artículos publicados a favor del método flipped classroom. Finalmente, el método flipped classroom se alza como una metodología docente que puede mejorar los resultados académicos y facilitar al estudiantado la autorregulación de su aprendizaje.
\end{abstract}

Descriptores: Calidad; Enseñanza superior; Eficacia; TIC; Mejora.

The peculiarity of the flipped classroom method lies in the change of learning roles, where the teacher acquires a secondary role as a guide and the student a primary role as an active agent. The trend of implementation in the stage of higher education is increasing and it is increasingly common to hear teaching experiences that revolve around its application in the classroom. Therefore, this paper aims to assess the effect of the flipped classroom methodology on the academic performance of university students from the studies indexed in the Wos and Scopus databases. A methodology of meta-analysis studies has been followed, with the purpose of measuring the size of the global and particular effect of the researchers and empirically verifying the gain in favour of the experimental or control group. The results show that the difference between groups is statistically significant and most of the studies confirm the increase in academic performance in the experimental group. However, there is evidence of a possible bias in the articles published in favour of the flipped classroom method. Finally, the flipped classroom method is raised as a teaching methodology that can improve academic results and make it easier for students to self-regulate their learning.

Keywords: Quality; Higher education; Effectiveness; ICT; Improvement.

*Contacto: tsola@ugr.es

ISSN: 1696-4713

www.rinace.net/reice/

revistas.uam.es/reice
Recibido: 1 de noviembre 2018

$1^{\text {a }}$ Evaluación: 20 de noviembre 2018

$2^{\text {a }}$ Evaluación: 27 de noviembre 2018

Aceptado: $\quad 30$ de noviembre 2018 


\section{Introducción}

El aprendizaje activo y la fijación por dar protagonismo al estudiantado se han consolidado como las principales premisas del aprendizaje del siglo XXI, a las que podemos añadir el uso de la tecnología. En este escenario, la metodología flipped classroom o aula invertida adquiere un especial énfasis, ya que aúna las principales tendencias educativas: el aprendizaje activo y uso de las TIC-Tecnologías de la Información y Comunicación (Hernández-Silva y Tecpan, 2017).

A este respecto, el Espacio Europeo de Educación Superior (EEES) inició el cambio de paradigma en los roles educativos, pasando de una docencia basada en la clase magistral a una docencia centrada en el alumnado (Reyes, 2015). En la misma línea, el avance constante de la tecnología ha incidido inevitablemente en el ámbito educativo, facilitando el acceso a la información a los estudiantes. Este hecho ha favorecido que el estudiante pueda autorregular su aprendizaje a partir del uso de dispositivos móviles (Aznar, Cáceres y Romero, 2018). Así pues, este trabajo tiene el objetivo de analizar el efecto de la metodología flipped classroom en el rendimiento académico de los estudiantes universitarios a partir de los estudios indexados en las bases de datos Wos y Scopus.

Es esencial, conocer, que tipo de influencia presenta el método flipped classroom en el rendimiento académico de los estudiantes. Cada día surgen nuevas experiencias de docentes que aplican este tipo de metodología en su aula, por lo que resulta esencial determinar el efecto que tiene en el aprendizaje. En este trabajo se aborda esta problemática desde una perspectiva metodológica de meta-análisis, la cual nos permite determinar el efecto global y particular de todas las investigaciones analizadas. La justificación y significación del estudio se ejemplifica en las tres preguntas de investigación que guían el trabajo:

- ¿Mejora el rendimiento académico de los estudiantes universitarios con una metodología de flipped classroom?

- ¿El tipo de materia en la que se implementa el flipped classroom y su duración determina su efecto positivo en el rendimiento académico?

- ¿Se publican principalmente artículos con resultados positivos de flipped classroom?

\section{Revisión de la literatura}

El flipped classroom se define como la inversión de roles en el aula, donde el estudiante adquiere un rol principal en su proceso de aprendizaje y el docente actúa como guía (Bergmann y Sams, 2012). Para ello, el temario es consultado fuera del horario lectivo por parte del estudiante y las horas destinadas a la docencia se dedican a la resolución de dudas (Castilla et al., 2015). Sin embargo, "invertir" el aula no constituye únicamente el hecho de que el estudiante pueda consultar la información previamente, sino que se trata de un enfoque integral de aprendizaje (Tourón y Santiago, 2015). En esta casuística, el docente suele preparar vídeos explicativos del temario y aportar material adicional para su estudio, mientras que el alumnado se involucra totalmente en su proceso de aprendizaje (Gonçalves y Quaresma, 2018). 
Las características asociadas a este método se corresponden con el aumento de las horas dedicadas al aprendizaje, el uso de la tecnología, protagonismo del alumnado, la inversión de roles y el compromiso por parte del docente y discente para llevar a cabo el proceso de aprendizaje (Miragall y García-Soriano, 2016). Por otra parte, el enfoque de flipped classroom permite acceder al contenido de forma ubicua, es decir, en cualquier momento y lugar gracias al uso de la tecnología, prescindiendo de la figura del profesor como transmisor de contenidos (Vidal et al., 2016). Se destacan así, diferentes ventajas: posibilidad de atender las diferencias individuales; favorece la autorregulación del aprendizaje y; genera un ambiente colaborativo en el aula (Aguilera et al., 2017; Bergmann y Sams, 2012; Castilla et al., 2015; Matzumura et al., 2018; Tourón y Santiago, 2015; Vidal et al., 2016). Mientras que como principales inconvenientes se destacan (Aguilera et al., 2017): reticencia del estudiantado; gran esfuerzo por parte del docente y el bajo grado de competencia digital que pueden poseer los estudiantes.

En relación a su efecto en el aprendizaje y en concreto atendiendo al constructo rendimiento académico, éste se define como el logro alcanzado por el estudiante en su proceso formativo. Son varios los estudios que relacionan el uso del método flipped classroom con el aumento del rendimiento académico de los estudiantes (Awidi y Paynter, 2018; Dehghanzadeh y Jafaraghaee, 2018; Domínguez et al., 2015; Hinojo et al., 2018; Matzumura et al., 2018) y con la mejora de los materiales educativos (Contreras et al., 2017). Esto destaca un foco de interés en torno a la temática y por la comprobación empírica de estos resultados, que a priori remarcan una mejora en las calificaciones de los estudiantes.

Siguiendo la línea de estudios previos de meta-análisis sobre flipped classroom, cabe mencionar los realizados por Cheng, Ritzhaupt y Antonenko (2018), Chen y otros (2018), Galindo (2018), Gillette y otros (2018), Hew y Lo (2018), Hu y otros (2018), Kang (2018). El estudio de Galindo (2018), centrado en la etapa de Educación Primaria, destaca que la materia donde más se aplica son las Matemáticas, el constructo más analizado es el rendimiento académico y el diseño metodológico con mayor uso el cuantitativo con pretest y postest. Por su parte, Chen y otros (2018), analizaron 46 estudios sobre flipped classroom (artículos y conferencias), en su mayoría centrados en el ámbito de las ciencias de la salud. A pesar de que la mayor parte de los trabajos muestra un efecto a favor del grupo experimental, el tamaño del efecto global no es significativo.

El meta-análisis realizado por Hew y Lo (2018) recoge 28 estudios empíricos de flipped classroom en ciencias de la salud. Los resultados muestran que el enfoque de aula invertida mejora significativamente el aprendizaje en el grupo experimental. El estudio de Hu y colaboradores (2018), se centró en los estudios de flipped classroom en enfermería. Los autores analizaron un total de 11 trabajos cuyos resultados muestran un efecto a favor del grupo que experimentó el método de aula invertida. Por su parte, Cheng, Ritzhaupt y Antonenko (2018) analizan el efecto general del flipped classroom a partir de un metaanálisis que recoge 55 publicaciones en diferentes ámbitos. Los resultados evidencian el tamaño del efecto global a favor del grupo experimental. También, el estudio de Kang (2018) recoge 36 estudios que compara los efectos del flipped classroom en escuelas coreanas. El tamaño del efecto global es significativo y a favor del grupo de aula invertida. Por último, Gillette y otros (2018), incluyen únicamente cinco artículos en su metaanálisis: cuatro de ellos con efecto a favor del grupo experimental y el restante a favor del grupo control. El tamaño del efecto global no muestra diferencias significativas entre los grupos. 
A diferencia de estos trabajos, la presente investigación se centra específicamente en la etapa de educación superior. Además, la búsqueda y recopilación de datos se ha ejecutado en bases de datos especializadas y con índice de impacto. Por último, los descriptores utilizados permiten no restringir la búsqueda con la finalidad de revisar un mayor número de documentos.

\section{Método}

El tipo de estudio realizado se enmarca dentro de la estrategia metodológica de los metaanálisis (Cristi, Solís y Rodríguez, 2016; Ferreira, Urrútia y Alonso-Coello, 2011; Kates, Wu y Coryn, 2018). El meta-análisis permite establecer el tamaño del efecto global de diversas investigaciones similares sobre una temática concreta, en este caso flipped classroom. Para asegurar la rigurosidad en la búsqueda de la bibliografía que compone la muestra del estudio, se han seguido los estándares de calidad de la declaración PRISMA referentes a los criterios de elegibilidad y la selección de los estudios (Urrútia y Bonfill, 2010).

Por otro lado, la búsqueda se ha realizado en las bases de datos con mayor impacto científico en las ciencias sociales (Hernández et al., 2013): Web Of Science (WOS) y Scopus. Asimismo, las palabras clave responden al tópico del trabajo, en este sentido, la ecuación de búsqueda implementada en ambas bases de datos correspondió a: "flipped classroom" AND "higher education".

Procedimiento de obtención de la muestra y variables de análisis

El procedimiento para la obtención de la muestra final se dividió en tres fases: búsqueda inicial de las palabras clave (fase 1); aplicación de los criterios de inclusión (a, b, c) y de exclusión ( $\mathrm{a}, \mathrm{b}$ ) en las opciones de filtrado de wos y Scopus (fase 2); revisión de todos los títulos, resúmenes y palabras clave para la aplicación de los criterios de inclusión (d, e, f) y de exclusión (c, d, e) (fase 3) (cuadro 1). Cabe resaltar que en wos la búsqueda tuvo lugar en los índices Social Sciences Citation Index (SSCI), Science Citation Index Expanded (SCIE) y Arts and Humanities Citation Index (AHCI). En resumen, el diagrama de flujo (figura 1), muestra la criba de la bibliografía hasta la obtención de la muestra definitiva $(n=12)$.

Cuadro 1. Criterios de inclusión y exclusión para el refinamiento de la muestra

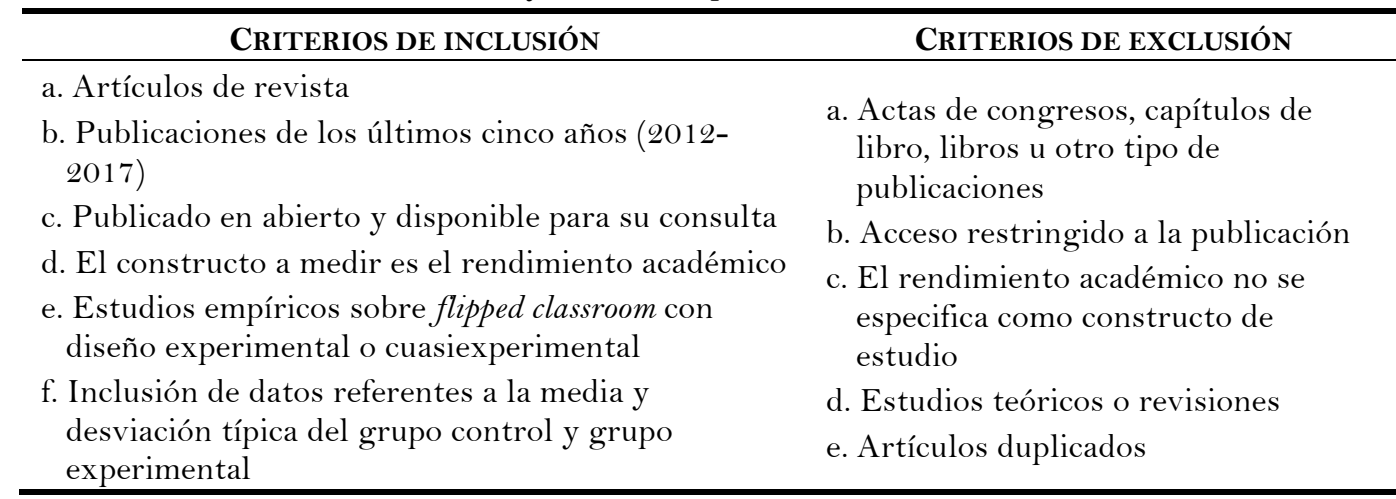

Fuente: Elaboración propia.

Posteriormente, se procedió a establecer las variables relacionadas con el constructo rendimiento académico en cada uno de los artículos. En base a estudios previos (Galindo, 2018; Gillette et al., 2018; Hew y Lo, 2018; Kang, 2018), se han detectado tres variables 
que pueden influir en las investigaciones que miden el rendimiento académico a partir del empleo del método flipped classroom y son fundamentales tener en cuenta en el metaanálisis: tipo de materia (V1), diseño metodológico (V2) y duración de los estudios (V3).

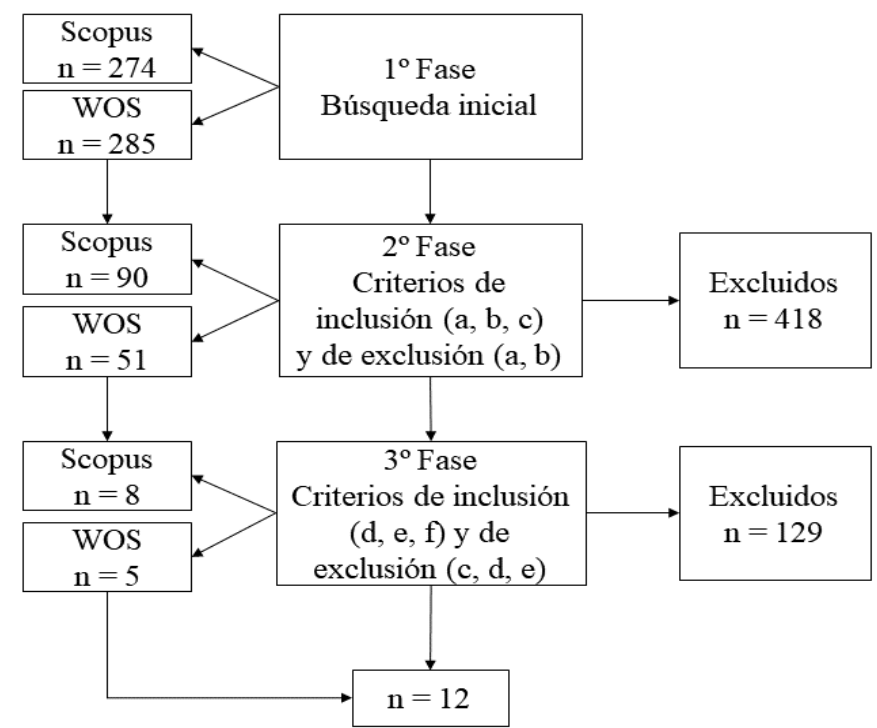

Figura 1. Diagrama de flujo del proceso seguido para la obtención de la muestra Fuente: Elaboración propia.

\section{Análisis de datos}

El análisis de datos se ha realizado a partir de la revisión de los artículos que componen la muestra, extrayendo la información referente a cada una de las variables por medio de una lectura pormenorizada de los documentos. Por otro lado, en el meta-análisis se ha empleado el software Review Manager, en su versión 5.3, para la confección del diagrama de bosque y gráfico de embudo (Chen et al., 2018; Cheng, Ritzhaupt y Antonenko, 2018; Gillette et al., 2018; Hew y Lo, 2018; Hu et al., 2018; Kang, 2018).

\section{Resultados}

Los estudios analizados se han focalizado en la introducción del flipped classroom en diferentes materias, por lo que nos encontramos con una gran variedad de campos de conocimiento. Entre ellos, destacan los ámbitos correspondientes a la química (15\%) y la física (15\%), donde se concentra un número superior de investigaciones (figura 2).

Por otro lado, los diseños metodológicos más utilizados en las investigaciones sobre flipped classroom son el diseño cuasiexperimental con grupo control (GC) y experimental (GE) y solo postest $(26,6 \%)$ y el diseño cuasiexperimental con GC y GE con pretest y postest $(26,6 \%)$. A este respecto, la mayor parte de estudios compara los datos obtenidos en un grupo con metodología tradicional y otro que ha seguido una metodología de flipped classroom, contrastando los resultados obtenidos en ambos grupos antes (únicamente en aquellos con pretest) y después. Otros diseños utilizados son: cuasiexperimental con dos GE y dos GC con pretest y postest (6,6\%); experimental con GC y GE con pretest y postest (6,6\%); cuasiexperimental con tres GC y tres GE con pretest y postest $(6,6 \%) \mathrm{y}$; experimental con GC y tres GE con pretest y postest $(6,6 \%)$. 


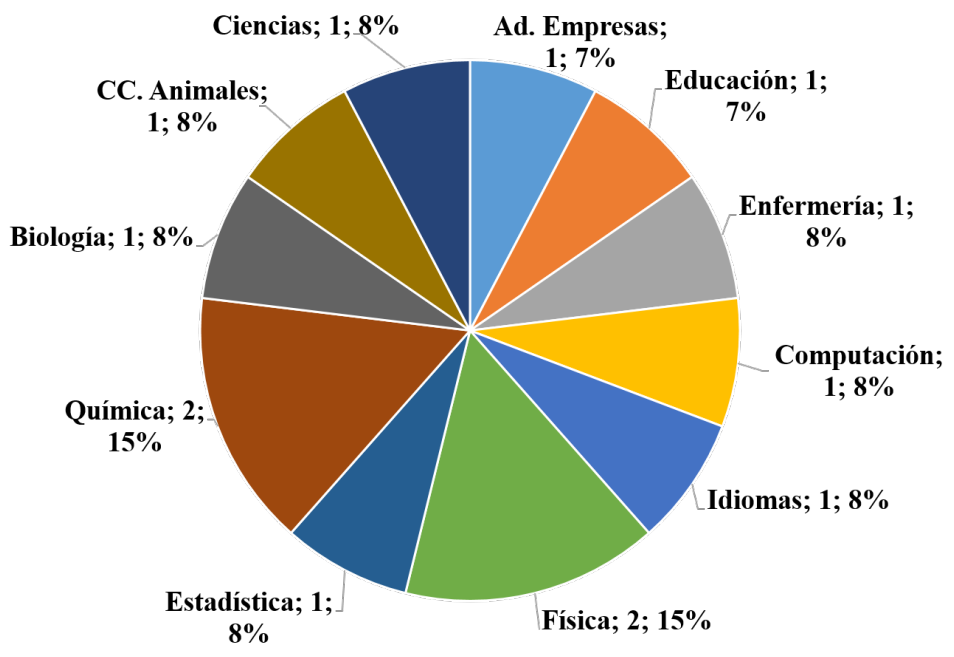

Figura 2. Materias objeto de estudio donde se ha implementado el método flipped classroom

Fuente: Elaboración propia.

En cuanto a la duración, varía de unas investigaciones a otras, oscilando entre un mínimo de seis y un máximo de 78 semanas $(\bar{x}=29, d t=19,3)$. La mayor parte comprende un periodo temporal de 26 semanas equivalentes a un semestre académico, que corresponde a la impartición de una materia concreta. Un tiempo suficiente para implementar de forma adecuada una metodología como el flipped classroom y poder comprobar sus efectos en el aprendizaje de los estudiantes.

Asimismo, nos encontramos con investigaciones divergentes en torno a tres supuestos: a favor del grupo control, a favor del grupo experimental y sin efecto. Cabe resaltar que en la confección del meta-análisis se han implementado todas las comparativas entre GC y GE: en Boevé y otros (2017) se contemplan las dos pruebas de recolección de datos del GC y GE (diferenciadas en el diagrama de bosque como grupo a y b); en Thai, De Weber y Valcke (2017) las tres comparativas entre el grupo de flipped classroom con el grupo tradicional (a), el de e-learning (b) y el de blended learning (c); Ryan y Reid (2016) los tres GC y tres GE (a, b y c); Mortensen y Nicholson (2015) las dos pruebas del GC y GE (a y b); Cohen y otros (2016) los dos GC y dos GE (a y b); y en Mattis (2015) las tres pruebas del GC y GE (a, b y c).

En suma, el tamaño del efecto (TE) global de las investigaciones analizadas se refleja en el diagrama de bosque (figura 3). La mayor parte de los estudios tienen un efecto positivo a favor del GE (Asiksoy y Ozdamli, 2017; Crimmins y Midkiff, 2017; Mattis, 2015-grupo b; Mennella, 2016; Mortensen y Nicholson, 2015; Sengel, 2016; Thai, De Weber y Valcke, 2017-grupo a y c), son todos aquellos que se sitúan a la derecha de la línea central, denominada línea de no efecto. La figura del diamante en el extremo derecho confirma que la diferencia entre grupos es estadísticamente significativa, confirmando que a priori el flipped classroom mejora el rendimiento académico de los estudiantes que fueron asignados al GE. De igual forma, el valor del TE $(p<0,00001)$ verifica la significación estadística de los datos. Los estudios que rozan la línea de no efecto, como su propio nombre indica, no presentan ningún efecto a favor del GC o GE (Boevé et al., 2017-grupo b y c; Cohen et al., 2016; Mattis, 2015-grupo a y c; Ryan y Reid, 2016; Thai, De Weber y Tsai et al., 2017; 
Valcke, 2017-grupo b; Wilcox, 2014). Por el contrario, solo un único estudio presenta un efecto a favor del GC (Boevé et al., 2017-grupo a).

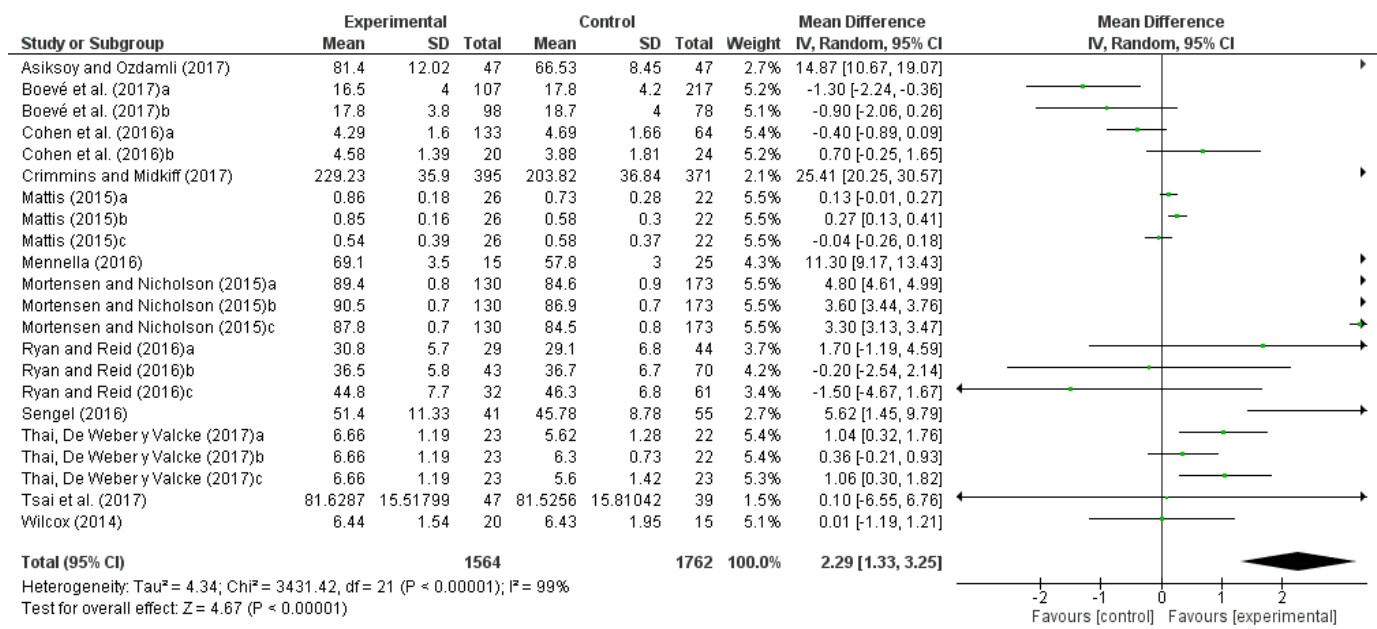

Figura 3. Diagrama de bosque del meta-análisis sobre las publicaciones de flipped classroom en educación superior que analizan el rendimiento académico Fuente: Elaboración propia.

Respecto al sesgo de publicación, el gráfico de embudo muestra la distribución de los estudios en torno al efecto (eje $\mathrm{X}$ ) y al tamaño (eje Y) (figura 4). La distribución heterogénea y la agrupación de las investigaciones en el lado izquierdo de la línea punteada indican que se han publicado mayoritariamente estudios con resultados positivos a favor del flipped classroom. La escasez de investigaciones que muestran lo contrario puede ser un indicador de un posible sesgo en los artículos que se publican sobre el efecto del flipped classroom. No obstante, a pesar de estas consideraciones, la situación de la mayor parte de los estudios en la parte superior refleja que se trata de trabajos precisos.

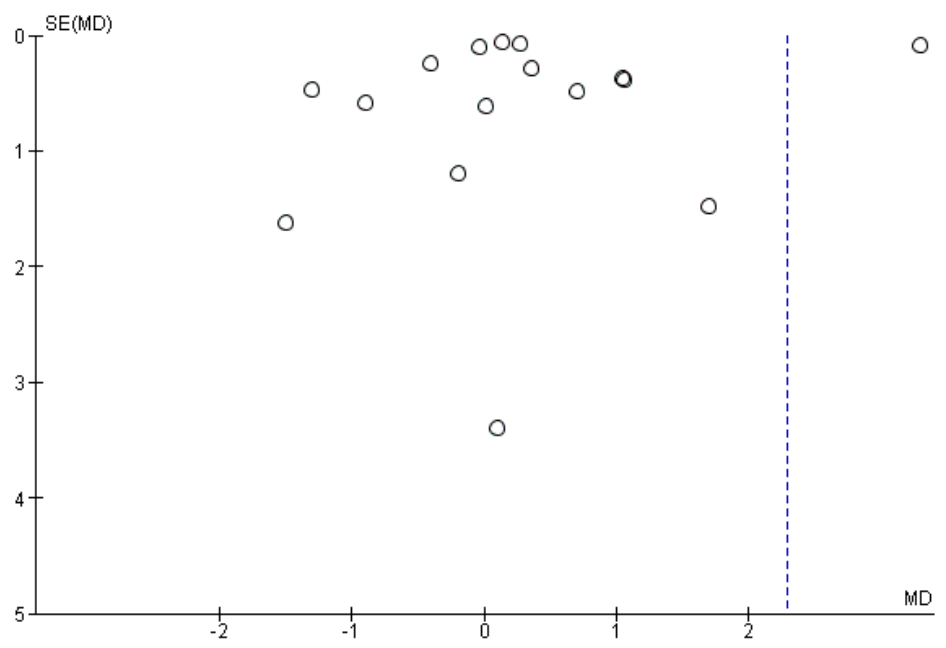

Figura 4. Gráfico de embudo sobre la distribución de los estudios analizados de flipped classroom

Fuente: Elaboración propia. 
A modo de resumen y con la finalidad de presentar los datos enlazados con cada uno de los estudios, la siguiente tabla especifica las correspondencias entre la materia, diseño, duración y efecto (cuadro 2 ).

Cuadro 2. Relación de cada estudio con las variables analizadas

\begin{tabular}{|c|c|c|c|c|}
\hline ESTUDIO & MATERIA & DISEÑO & DURACIÓN* & EFECTO \\
\hline Aşıksoy y Ozdamli (2017) & Física & $\begin{array}{l}\mathrm{R} \mathrm{GC} \mathrm{O}_{1}-\mathrm{O}_{2} \\
\mathrm{R} \mathrm{GE} \mathrm{O}_{1} \mathrm{X} \mathrm{O}_{2}\end{array}$ & 10 & GE \\
\hline Boevé y otros (2017) & Estadística & $\begin{array}{l}\mathrm{GC}-\mathrm{O}_{2} \\
\mathrm{GE} \times \mathrm{O}_{2}\end{array}$ & 26 & $\mathrm{GC} / \mathrm{N}$ \\
\hline Cohen y otros (2016) & $\begin{array}{l}\text { Ad. Empresas / } \\
\text { Educación }\end{array}$ & $\begin{array}{l}2 \mathrm{GCO}_{1}-\mathrm{O}_{2} \\
2 \mathrm{GE} \mathrm{O}_{1} \mathrm{X} \mathrm{O}_{2}\end{array}$ & $78 / 52$ & $\mathrm{~N}$ \\
\hline Crimmins y Midkiff (2017) & Química & $\begin{array}{l}\mathrm{GC}-\mathrm{O}_{2} \\
\mathrm{GE} \times \mathrm{O}_{2} \\
\end{array}$ & 52 & GE \\
\hline Mattis (2015) & Enfermería & $\begin{array}{l}\mathrm{GC} \mathrm{O}_{1}-\mathrm{O}_{2} \\
\mathrm{GE} \mathrm{O}_{1} \mathrm{X} \mathrm{O}_{2}\end{array}$ & 26 & $\mathrm{GE} / \mathrm{N}$ \\
\hline Mennella (2016) & Biología & $\begin{array}{l}\mathrm{GC}-\mathrm{O}_{2} \\
\mathrm{GE} \times \mathrm{O}_{2} \\
\end{array}$ & 26 & GE \\
\hline $\begin{array}{l}\text { Mortensen y Nicholson } \\
(2015)\end{array}$ & CC. Animales & $\begin{array}{l}\mathrm{GC} \mathrm{O}_{1}-\mathrm{O}_{2} \\
\mathrm{GE} \mathrm{O}_{1} \mathrm{X} \mathrm{O}_{2}\end{array}$ & 15 & GE \\
\hline Ryan y Reid (2015) & Química & $\begin{array}{l}3 \mathrm{GC} \mathrm{O}_{1}-\mathrm{O}_{2} \\
3 \mathrm{GE} \mathrm{O}_{1} \mathrm{X} \mathrm{O}_{2} \\
\end{array}$ & 26 & $\mathrm{~N}$ \\
\hline Sengel (2016) & Física & $\begin{array}{l}\mathrm{GC} \mathrm{O}_{1}-\mathrm{O}_{2} \\
\mathrm{GE} \mathrm{O}_{1} \times \mathrm{O}_{2} \\
\end{array}$ & 12 & GE \\
\hline $\begin{array}{l}\text { Thai, De Weber y Valcke } \\
(2017)\end{array}$ & Ciencias & $\begin{array}{l}\text { R } 1 \mathrm{GC} \mathrm{O}_{1}-\mathrm{O}_{2} \\
\mathrm{R} 3 \mathrm{GE} \mathrm{O}_{1} \times \mathrm{O}_{2} \\
\end{array}$ & 6 & $\mathrm{GE} / \mathrm{N}$ \\
\hline Tsai y otros (2017) & Computación & $\begin{array}{l}\mathrm{GC}-\mathrm{O}_{2} \\
\mathrm{GE} \times \mathrm{O}_{2} \\
\end{array}$ & 26 & $\mathrm{~N}$ \\
\hline Wilcox (2014) & Idiomas & $\begin{array}{l}\mathrm{GC} \mathrm{O}_{1}-\mathrm{O}_{2} \\
\mathrm{GE} \mathrm{O}_{1} \mathrm{X} \mathrm{O}_{2} \\
\end{array}$ & 26 & $\mathrm{~N}$ \\
\hline
\end{tabular}

Nota: ${ }^{*}$ Duración en semanas. $\mathrm{R}=$ Aleatorización. $\mathrm{O}=$ Test. $\mathrm{GE}=$ Grupo experimental. $\mathrm{GC}=$ Grupo control. $\mathrm{X}=$ Tratamiento. $\mathrm{N}=$ No efecto.

Fuente: Elaboración propia.

\section{Discusión y conclusiones}

Los datos muestran un amplio abanico de ámbitos de conocimiento donde se implementa el flipped classroom en educación superior. No obstante, predomina su introducción en aquellas materias relacionadas con las ciencias puras, coincidiendo con los resultados del meta-análisis de Galindo (2018). En la misma línea, el diseño metodológico con mayor prevalencia es el cuasiexperimental con uno o varios grupos control y experimental y pretest y postest.

La variabilidad de campos de conocimiento resalta la inquietud docente por mejorar el aprendizaje de los estudiantes. Además, es un indicador de que docentes de distintas disciplinas conocen los beneficios asociados al método flipped classroom (Aguilera et al., 2017; Bergmann y Sams, 2012; Castilla et al., 2015; Matzumura et al., 2018; Tourón y Santiago, 2015; Vidal et al., 2016). Este enfoque integral del aprendizaje, que sigue las premisas del EEES (Reyes, 2015), constituye un potente recurso para que el estudiante pueda autorregular y coger las riendas de su proceso de aprendizaje (Tourón y Santiago, 
2015). Cualidades que hoy en día son necesarias para afrontar con éxito demandas personales, sociales y profesionales.

En relación a la duración de las investigaciones, la mayor parte se concentra en la experimentación del flipped classroom durante un semestre, dentro de una materia concreta. A raíz de los resultados resulta óptima la duración, puesto que se obtienen datos similares en periodos temporales más amplios (un año académico) y menos duraderos (6 semanas).

Centrándonos en el constructo de análisis, rendimiento académico, se confirma que el tamaño del efecto global de los 12 estudios analizados es a favor del grupo experimental. Por lo que se puede afirmar que el flipped classroom implica una mejora del rendimiento académico de los estudiantes (Awidi y Paynter, 2019; Dehghanzadeh y Jafaraghaee, 2018; Domínguez et al., 2015; Hinojo et al., 2018; Matzumura et al., 2018). Por otro lado, a diferencia del meta-análisis de Chen y otros (2018) y Gillette y colaboradores (2018), se constata la diferencia significativa entre el grupo control y experimental. En la misma línea que los trabajos de Cheng, Ritzhaupt y Antonenko (2018), Hew y Lo (2018), Hu y otros (2018) y Kang (2018).

Atendiendo al gráfico de embudo, es esencial contemplar el posible sesgo en las publicaciones. Es una práctica habitual que los investigadores solo publiquen los datos positivos y desechen los resultados negativos. En este caso, mayoritariamente los estudios muestran datos empíricos que avalan los beneficios del método flipped classroom. Nos encontramos así, con dos supuestos: el flipped classroom presenta una elevada tasa de éxito en el aumento del rendimiento académico de los estudiantes o existe una escasez de publicaciones que demuestren lo contrario.

Por último, destacar, la respuesta a las diferentes cuestiones planteadas que han sido la base del estudio de meta-análisis:

¿Mejora el rendimiento académico de los estudiantes universitarios con una metodología de flipped classroom?

Los datos de los distintos estudios, reflejados en el diagrama de bosque, muestran que el rendimiento académico mejora en aquellos grupos experimentales donde se ha implementado el método flipped classroom. Concretamente en los estudios de Mattis (2015), Mortensen y Nicholson (2015), Mennella (2016), Sengel (2016), Asiksoy y Ozdamli (2017), Crimmins y Midkiff (2017) y Thai, De Weber y Valcke (2017). Al igual, la mayor parte de los estudios previos de meta-análisis recogen la mejora del aprendizaje en aquellos estudiantes que han seguido una metodología basada en el flipped classroom (Cheng, Ritzhaupt y Antonenko, 2018; Hew y Lo, 2018; Hu et al., 2018; Kang, 2018).

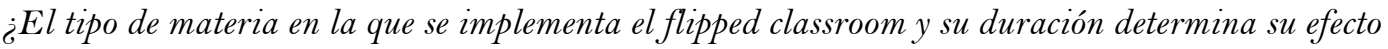
positivo en el rendimiento académico?

La materia y duración no determina el efecto positivo. Nos encontramos con estudios centrados en materias como química, con una duración anual (Crimmins y Midkiff, 2017), que presenta el mismo efecto a favor del grupo experimental que otros en ciencias de los animales y con una duración de 15 semanas (Mortensen y Nicholson, 2015). Ocurre lo mismo con la variabilidad de ámbitos de aplicación de estudios previos que muestran un efecto similar en enfermería (Hu et al., 2018), ciencias de la salud (Chen et al., 2018; Hew y Lo, 2018) e incluso en escuelas (Kang, 2018). 
¿Se publican principalmente artículos con resultados positivos de flipped classroom?

A priori se podría confirmar esta premisa, puesto que son más numerosos. No obstante, habría que tener en consideración otros factores como que el flipped classroom obtenga una elevada tasa de éxito. Cabe plantearse esta posibilidad, puesto que son numerosos los trabajos empíricos que muestran un efecto positivo a su favor (Asiksoy y Ozdamli, 2017; Crimmins y Midkiff, 2017; Mattis, 2015; Mennella, 2016; Mortensen y Nicholson, 2015; Sengel, 2016; Thai, De Weber y Valcke, 2017), así como distintos meta-análisis (Cheng, Ritzhaupt y Antonenko, 2018; Hew y Lo, 2018; Hu et al., 2018; Kang, 2018).

Así pues, la respuesta a las distintas cuestiones también implica el cumplimiento del objetivo planteado acerca de: analizar el efecto de la metodología flipped classroom en el rendimiento académico de los estudiantes universitarios a partir de los estudios indexados en las bases de datos wos y Scopus. Cabe resaltar que las principales limitaciones metodológicas de este trabajo se relacionan con la búsqueda en las bases de datos consultadas (WOS y Scopus). En esta línea, se han prescindido de los artículos indexados en otras bases de datos que podrían obtener la información objeto de estudio. Sin embargo, creemos pertinente consultar en un primer momento los artículos indexados en las bases con mayor impacto científico. Además, aquellos estudios que no presentaban los datos referentes a la media de grupos (control y experimental) y desviación típica se han eliminado, por no aportar la información clave para la realización del meta-análisis.

Finalmente, este estudio sienta las bases de diferentes prospectivas de trabajo, entre ellas: continuidad temporal del meta-análisis en años venideros; ampliación del meta-análisis consultando en otras bases de datos científicas y otras etapas educativas; experimentación del método flipped classroom para contribuir al cuerpo de conocimiento sobre sus efectos en el rendimiento académico y, a partir de la constatación empírica de los resultados a favor del grupo experimental, los docentes tienen datos fiables de éxito del flipped classroom para su implementación en el aula.

\section{Referencias}

Aguilera, C., Manzano, A., Martínez, I., Lozano, M. C. y Casiano, C. (2017). El modelo flipped classroom. International Journal of Developmental and Educational Psychology, 4(1), 261-266. https://doi.org/10.17060/ijodaep.2017.n1.v4.1055

Asiksoy, G. y Ozdamli, F. (2017). The flipped classroom approach based on the 5e learning cycle model. Croatian Journal of Education, 19(4), 1131-1166. https://doi.org/10.15516/cje.v19i4.2564

Awidi, I. T. y Paynter, M. (2018). The impact of a flipped classroom approach on student learning $\begin{array}{lllll}\text { experience. } & \text { Computers } & \mathcal{E}^{\circ} & \text { Education, } & \text { 128, 269-283. }\end{array}$ https://doi.org/10.1016/j.compedu.2018.09.013

Aznar, I., Cáceres, M. P. y Romero, J. M. (2018). Indicadores de calidad para evaluar buenas prácticas docentes de mobile learning en educación superior. Education in the Knowledge Society (EKS), 19(3), 53-68. https://doi.org/10.14201/eks20181935368

Bergmann, J. y Sams, A. (2012). Flip your classroom: Reach every student in every class every day. Eugene, OR: International Society for Technology in Education.

Boevé, A. J., Meijer, R. R., Bosker, R. J., Vugteveen, J., Hoekstra, R. y Albers, C. J. (2017). Implementing the flipped classroom: An exploration of study behavior and student 
performance. Higher Education, 74, 1015-1032. https://doi.org/10.1007/s 10734-016-0104y

Castilla, G., Alriols, J., Romana, M. y Escribano, J. J. (2015). Resultados del estudio experimental de flipped learning en el ámbito de la enseñanza de matemáticas en ingeniería. En M. A. Ruiz Rosillo (Coord.), Actas de las XII Jornadas Internacionales de Innovación Universitaria (pp. 774-782). Madrid: Universidad Europea de Madrid.

Chen, K. S., Monrouxe, L., Lu, Y. H., Jenq, C. C., Chang, Y. J., Chang, Y. C. y Yee-Chee, P. (2018). Academic outcomes of flipped classroom learning: A meta-analysis. Medical Education, 52, 910-924. https://doi.org/10.1111/medu.13616

Cheng, L., Ritzhaupt, A. D. y Antonenko, P. (2018). Effects of the flipped classroom instructional strategy on students' learning outcomes: A meta-analysis. Educational Technology Research and Development, 66(6), 1-32. https://doi.org/10.1007/s11423-018-9633-7

Cohen, M. E., Poggiali, J., Lehner-Quam, A., Wright, R. y West, R. K. (2016). Flipping the classroom in business and education one-shot sessions: A research study. Journal of Information Literacy, 10(2), 40-63. https://doi.org/10.11645/10.2.2127

Contreras, J. A., Arias, J., Melo, M. G. y Martín, R. (2017). Uso del modelo de aprendizaje inverso para mejorar materiales educativos universitarios. Revista lbérica de Sistemas y Tecnologías de Información, 23, 17-32. https://doi.org/10.17013/risti.23.17-32

Crimmins, M. T. y Midkiff, B. (2017). High structure active learning pedagogy for the teaching of organic chemistry: Assessing the impact on academic outcomes. Journal of Chemical Education, 94, 429-438. https://doi.org/10.1021/acs.jchemed.6b00663

Cristi, C., Solís, P. y Rodríguez, F. (2016). Consideraciones previas a un metaanálisis. Nutrición Hospitalaria, 33(2), 510-511. https://doi.org/10.20960/nh.533

Dehghanzadeh, S. y Jafaraghaee, F. (2018). Comparing the effects of traditional lecture and flipped classroom on nursing students' critical thinking disposition: A quasi-experimental study. Nurse Education Today, 71, 151-156. https://doi.org/10.1016/j.nedt.2018.09.027

Domínguez, L. C., Vega, N. V., Espitia, E. L., Sanabria, A. E., Corso, C., Serna, A. M. y Osorio, C. (2015). Impacto de la estrategia de aula invertida en el ambiente de aprendizaje en cirugía: Una comparación con la clase magistral. Biomédica, 35, 513-521. https://doi.org/10.7705/biomedica.v35i4.2640

Ferreira, I., Urrútia, G. y Alonso-Coello, P. (2011). Revisiones sistemáticas y metaanálisis: Bases conceptuales e interpretación. Revista Española de Cardiología, 64(8), 688-696. https://doi.org/10.1016/j.recesp.2011.03.029

Galindo, H. (2018). Un meta-análisis de la metodología flipped classroom en el aula de educación primaria. EDUTEC. Revista Electrónica de Tecnología Educativa, 63, 73-85. https://doi.org/10.21556/edutec.2018.63.983

Gillette, C., Rudolph, M., Kimble, C., Rockich-Winston, N., Smith, L. y Broedel-Zaugg, K. (2018). A meta-analysis of outcomes comparing flipped classroom and lecture. American Journal of Pharmaceutical Education, 82(5), 433-440. https://doi.org/10.5688/ajpe6898

Gonçalves, Z. T. y Quaresma, D. (2018). Metodologia ativa: Sala de aula invertida e suas práticas na educação básica. REICE. Revista Iberoamericana sobre Calidad, Eficacia y Cambio en Educación, 16(4), 63-78. https://doi.org/10.15366/reice2018.16.4.004

Hernández, V., Reverter, J., Jové, M. C. y Mayolas, M. C. (2013). Hábitos de publicación de profesores de los departamentos de didáctica de la expresión corporal y educación física de las universidades públicas españolas. European Journal of Human Movement, 30, 1-12. 
Hernández-Silva, C. y Tecpan, S. (2017). Aula invertida mediada por el uso de plataformas virtuales: Un estudio de caso en la formación de profesores de física. Estudios Pedagógicos, 18(3), 193-204. https://doi.org/10.4067/S0718-07052017000300011

Hew, K. F. y Lo, C. K. (2018). Flipped classroom improves student learning in health professions education: A meta-analysis. BMC Medical Education, 18(38), 1-12. https://doi.org/10.1186/s 12909-018-1144-Z

Hinojo, F. J., Mingorance, A. C., Trujillo, J. M., Aznar, I. y Cáceres, M. P. (2018). Incidence of the flipped classroom in the physical education students' academic performance in university contexts. Sustainability, 1O(5), 1-13. https://doi.org/10.3390/su10051334

Hu, R., Gao, H., Ye, Y., Ni, Z., Jiang, N. y Jiang, X. (2018). Effectiveness of flipped classrooms in Chinese baccalaureate nursing education: A meta-analysis of randomized controlled trials. $\begin{array}{lllll}\text { International Journal of Nursing } & \text { Studies, } & 79, & \text { 94-103. }\end{array}$ https://doi.org/10.1016/j.ijnurstu.2017.11.012

Kang, S. (2018). The comparison of flipped learning effects in Korean school classes. Journal of Advanced Research in Dynamical and Control Systems, 10(1), 100-108.

Kates, A. W., Wu, H. y Coryn, C. (2018). The effects of mobile phone use on academic performance: A metaanalysis. Computers $\Theta^{\circ}$ Education, 127, 107-112. https://doi.org/10.1016/j.compedu.2018.08.012

Mattis, K. V. (2015). Flipped classroom versus traditional textbook instruction: Assessing accuracy and mental effort at different levels of mathematical complexity. Technology, Knowledge and Learning, 20, 231-248. https://doi.org/10.1007/s 10758-014-9238-0

Matzumura, J. P., Gutiérrez, H., Zamudio, L. A. y Zavala, J. C. (2018). Aprendizaje invertido para la mejora y logro de metas de aprendizaje en el curso de metodología de la investigación en estudiantes de universidad. Revista Electrónica Educare, 22(3), 1-21. https://doi.org/10.15359/ree.22-3.9

Mennella, T. (2016). Comparing the efficacy of flipped vs. alternative active learning in a college genetics course. The American Biology Teacher, 78(6), 471-479.

Miragall, M. y García-Soriano, G. (2016). Transformando una clase del grado en psicología en una flipped classroom. @TIC. Revista d'Innovació Educativa, 17, 21-29. https://doi.org/10.7203/attic.17.9097

Mortensen, C. J. y Nicholson, A. M. (2015). The flipped classroom stimulates greater learning and is a modern 21 st century approach to teaching today's undergraduates. Journal of Animal Science, 93, 3722-3731. https://doi.org/10.2527/jas2015-9087

Reyes, A. E. (2015). Educación y formación en la Unión Europea: Análisis del proceso de Bolonia, el espacio europeo de educación superior, la estrategia Europa 2020 y el programa Erasmus+. Derecho y Cambio Social, 12(42), 1-23.

Ryan, M. D. y Reid, S. A. (2016). Impact of the flipped classroom on student performance and retention: A parallel controlled study in general chemistry. Journal of Chemical Education, 93, 13-23. https://doi.org/10.1021/acs.jchemed.5boo717

Sengel, E. (2016). To FLIP or not to FLIP: Comparative case study in higher education in Turkey. Computers in Human Behavior, 64, 547-555. https://doi.org/10.1016/j.chb.2016.07.034

Thai, N. T., De Weber, B. y Valcke, M. (2017). The impact of a flipped classroom design on learning performance in higher education: Looking for the best "blend" of lectures and guiding questions with feedback. Computers and Education, 107, 113-126. https://doi.org/10.1016/j.compedu.2017.01.003 
Tourón, J. y Santiago, R. (2015). El modelo flipped learning y el desarrollo del talento en la escuela. Revista de Educación, 368, 196-231. https://doi.org/10.4438/1988-592X-RE-2015-368-288

Tsai, C. W., Shen, P. D., Chiang, Y. C. y Lin, C. H. (2017). How to solve students' problems in a flipped classroom: A quasi-experimental approach. Universal Access in the Information Society, 16, 225-233. https://doi.org/10.1007/s 10209-016-0453-4

Urrútia, G. y Bonfill, X. (2010). Declaración PRISMA: Una propuesta para mejorar la publicación de revisiones sistemáticas y metaanálisis. Medicina Clínica, 135(11), 507-511. https://doi.org/10.1016/j.medcli.2010.01.015

Vidal, M., Rivera, N., Nolla, N., Morales, I. R. y Vialart, M. N. (2016). Aula invertida, nueva estrategia didáctica. Educación Médica Superior, 30(3), 1-12.

Wilcox, A. (2014). Information literacy and the flipped classroom: Examining the impact of a oneshot flipped class on student learning and perceptions. Communications in Information Literacy, 8(2), 225-235. https://doi.org/10.15760/comminfolit.2014.8.2.168

\section{Breve CV de los autores}

\section{Tomás Sola Martínez}

Catedrático de Didáctica y Organización Escolar (DOE) de la Universidad de Granada (UGR). Doctor en Ciencias de la Educación por esta misma Universidad. Su trayectoria investigadora se centra en la Educación Especial y la atención a la diversidad. Es autor de innumerables libros y artículos científicos relacionados con el área. Investigador de diferentes proyectos y contratos de investigación, financiados en convocatorias competitivas a nivel autonómico, nacional e internacional. Actualmente es miembro del Grupo de Investigación "Análisis de la Realidad EducativA", AREA (HUM672) del PAIDI de la Junta de Andalucía. ORCID ID: https://orcid.org/O000-0001-9471-1249. Email: tsola@ugr.es

\section{Inmaculada Aznar Díaz}

Profesora Titular de Universidad del Departamento de Didáctica y Organización Escolar de la Universidad de Granada. Doctora en Ciencias de la Educación por la misma Universidad, Licenciada en Psicopedagogía y Maestra de Educación Primaria. Trabaja en la línea de organización escolar, competencia digital en educación, la formación para el empleo y las metodologías activas para el aprendizaje con TIC. Autora de varios libros, capítulos y artículos en revistas científicas internacionales relacionadas con el área y dichas líneas de investigación. Coordinadora de diferentes proyectos de innovación educativa y buenas prácticas docentes aprobados por la Agencia Nacional de Evaluación de la Calidad y Acreditación (ANECA). ORCID ID: https://orcid.org/0000-0002-00181150.Email: iaznar@ugr.es

\section{José María Romero Rodríguez}

Personal Docente e Investigador en el Departamento de Didáctica y Organización Escolar de la Universidad de Granada. Graduado en Pedagogía por esta misma Universidad, habiendo obtenido el Premio Extraordinario Fin de Grado. Posteriormente, ha cursado el Máster Universitario en Investigación e Innovación en Currículum y Formación, especialidad en Didáctica y Organización Escolar. En la actualidad cursa los estudios de doctorado en el programa de Ciencias de la Educación. Desempeña su labor investigadora 
dentro del grupo de investigación AREA /HUM-672). Es autor de varias comunicaciones y artículos científicos sobre el uso de las Tecnologías de la Información y Comunicación (TIC) para la mejora de la enseñanza y el aprendizaje, en concreto desarrolla su línea en torno al mobile learning y tecnologías móviles emergentes. ORCID ID: https://orcid.org/o000-0002-9284-8919. Email: romejo@ugr.es

\section{Antonio-Manuel Rodríguez-García}

Licenciado en Pedagogía. Máster de Investigación e Innovación en Currículum y Formación (Universidad de Granada) y Máster en Innovación y Gestión del Conocimiento (Universidad de Málaga). Profesor Sustituto Interino en el Departamento de Didáctica y Organización (Campus de Ceuta) de la Universidad de Granada. Líneas principales de investigación: Tecnologías de la Información y la Comunicación, formación y metodología docente, Educación Superior, la formación por competencias, la innovación, entre otras. Todo ello ha dado como fruto la participación y publicación de trabajos en diversas revistas, congresos, jornadas...así como la realización de diferentes estancias de investigación y docencia en países como Portugal, República Checa o Ucrania. ORCID ID: https://orcid.org/0000-0003-3394-2777. Email: arodrigu@ugr.es 